\title{
Sิำ $\mathfrak{a} \mathfrak{l t}$.
}

Eeite

1. Sinterlaffere Sragmente fum Jwenten Ibeil bes laofoon.

3

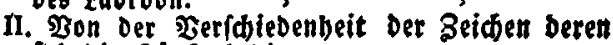
fich bie Sunfte beoienen. III. Die veríchiebenen Dimenfionen ictwächen Die 2 irtung ber Malerey. $\quad 62$ IV. FTleinere fragmente artififon Subalts, welche bey Der zwenten 2ub́gabe des faoloons fion als anbang be Eant gemadt worden finb.

1 Allegorie.

2. Fon Den notbwenbigen Fehlern.

3. Heber eine Etelle aub 3 inelmanm $7 \mathbf{I}$ fabiate den Benodorus betreffend.

Heber einige Stelley aus Dem mont

5. Ueber etne Strlle aus Dem Potter

6. Ron einem per,pectivifhen Sleichniffe Des Squmers

7. Eingelne Bedanten zur Fortfézung Des Saotoons

8. Leber Berarbs Meinung, das die Males rey auch das Erbabene ausorícten tỏnne, melases mit Der Ordóse Det Dimenfionen ver: bunden iff. 


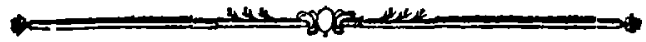

9. Einige Bemerfungen aus ben Obfervations fur l'Italie, Tom II und Richardfon's Traité de la peinture, T. I. '

V. Wie bie Alten Den $\mathfrak{x} 00$ gebilbet. 103

VI. Heber Die fo genannte Agrippine, unter Den 2 Iterthumern fu DreBden 226

VII. Anmerlungen fu winfelmants (s) ( hidid) te Der funf́ bes altertl)umb.

VIII. Heber Die Albnenbiloer Der Romer. Eine antiquarifoe unteriudung.

IX. Fragment úber bie Sflifhe safel.

1. Befकichte Der Ifichen aafel

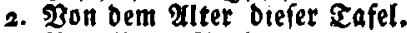

3. 2on thren auslegern

4. Einige Merfmúroigfeiten biefer $\mathfrak{z a f e l . ~} 34 \mathrm{I}$. X. Ileinere antiquarifoe fragmente.

1. Tarnatiben.

2. Diosforibes.

3. Brottesten

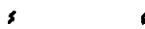

- 366

327

4. Heber die mängel bes antiquarijoen' $\mathrm{Stus}$ biumb. 


\section{I. \\ $\mathfrak{A} \mathfrak{x} \mathfrak{i} \mathfrak{t} \mathfrak{i} \mathfrak{e}$ \\ uno \\ antiquarif(de Sdriften. \\ (Jortergung.)}

Decrn. Edr. X. xb.

$\boldsymbol{x}$ 
